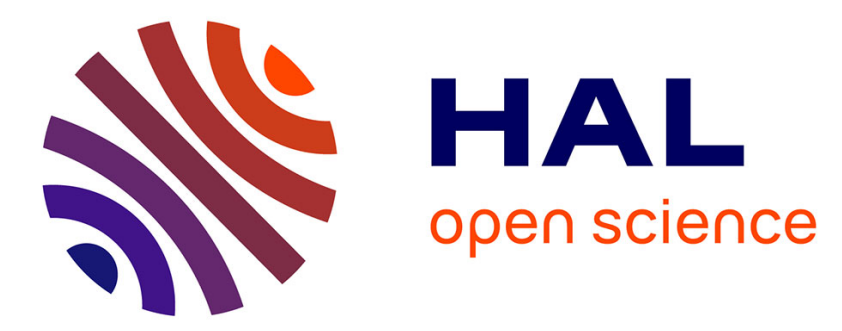

\title{
La communication d'informations au cours des ventes aux enchères de bois par adjudication: ce que dit la théorie économique
}

Vianney Dequiedt

\section{- To cite this version:}

Vianney Dequiedt. La communication d'informations au cours des ventes aux enchères de bois par adjudication: ce que dit la théorie économique. Revue forestière française, 2006, 58 (5), pp.478-482. $10.4267 / 2042 / 6772$. hal-03449584

\section{HAL Id: hal-03449584 \\ https://hal.science/hal-03449584}

Submitted on 25 Nov 2021

HAL is a multi-disciplinary open access archive for the deposit and dissemination of scientific research documents, whether they are published or not. The documents may come from teaching and research institutions in France or abroad, or from public or private research centers.
L'archive ouverte pluridisciplinaire HAL, est destinée au dépôt et à la diffusion de documents scientifiques de niveau recherche, publiés ou non, émanant des établissements d'enseignement et de recherche français ou étrangers, des laboratoires publics ou privés. 


\section{LIBRE EKPRESSION}

\section{LA COMMUNICATION D'INFORMATIONS AU COURS DES VENTES AUX ENCHÈRES DE BOIS PAR ADJUDICATION : CE QUE DIT LA THÉORIE ÉCONOMIQUE}

L'objectif de cette note est de proposer un éclairage théorique sur les effets de la communication publique d'informations au cours des ventes aux enchères de bois par adjudication.

Une telle communication est au cœur de débats animés entre d'une part les acheteurs souhaitant des procédures de vente transparentes et favorisant l'émergence d'un prix de marché et d'autre part les vendeurs souhaitant garantir la compétition entre les acheteurs.

La théorie économique met en lumière les nombreux effets qui sont induits par la communication d'informations au cours d'une vente et distingue des situations dans lesquelles plus d'information est favorable ou défavorable à l'efficacité de la vente. La théorie économique permet donc de préciser les arguments des uns et des autres et de fournir les concepts nécessaires à la discussion.

De manière préliminaire, il convient de rappeler le rôle général des procédures d'enchères. Dans un contexte de rareté des ressources et en faisant abstraction des enjeux de redistribution, l'efficacité économique (1) requiert que chaque ressource soit attribuée à l'acheteur qui lui accorde la plus grande valeur. Les procédures d'enchères, en mettant en concurrence les différents acheteurs, sont généralement reconnues pour avoir de bonnes propriétés en termes d'efficacité économique. De fait, les mécanismes d'enchères sont utilisés pour vendre de nombreux biens (bons du trésor, fleurs, œuvres d'art, vins, poissons, ou encore électricité) et le développement d'internet a rendu leur utilisation aisée (on peut citer le succès de sites comme e-bay). Dans le cas de la filière-bois, utiliser des enchères pour vendre le bois se veut donc un moyen d'assurer que le bois est vendu à celui qui en fera le meilleur usage, au bénéfice de l'ensemble de la filière.

Il est également important de souligner que l'existence de procédures d'enchères est étroitement liée à l'existence d'asymétries d'information. Par le terme asymétries d'information, il s'agit de signifier le fait que tous les acteurs économiques ne disposent pas de toute l'information souhaitable, et n'ont surtout pas accès à la même information. Si l'environnement économique était parfaitement connu de tous, alors l'allocation efficace du bois ne nécessiterait pas de tels mécanismes. Le choix du meilleur partenaire commercial et un prix fixé au cas par cas feraient l'affaire.

La note est organisée comme suit. Nous introduisons tout d'abord les concepts de signaux, type, valeur privée et valeur commune. Puis, nous décrivons succinctement les ventes aux enchères de bois par adjudication telles qu'elles sont organisées par l'ONF et explicitons ce que nous enten-

(1) La notion d'efficacité utilisée par les économistes est définie à l'aide du critère de Pareto [du nom de l'économiste italien Vilfredo Pareto (1848-1923)]. Selon ce critère, une situation est dite efficace s'il n'est pas possible de la modifier sans détériorer la position d'au moins l'un des acteurs. 
dons par communication de signaux au cours des ventes. Ensuite, nous proposons une grille de lecture pour évaluer les impacts des différents signaux, en caractérisant l'information transmise par ces signaux et son impact sur les stratégies des acheteurs. Enfin, nous soulignons quelques éléments centraux pour l'évaluation de la pertinence des signaux.

\section{QUELQUES DÉFINITIONS UTILES}

L'information est la notion centrale de l'analyse économique des enchères. Que l'on parle d'information privée des acheteurs, d'agrégation d'information dans le prix de vente ou de communication d'information au cours de la vente, le même terme revient toujours. Pour plus de clarté, il est utile de distinguer les différents emplois du mot information. Nous parlerons de communication de signaux pour désigner toute information délivrée par l'organisateur de la vente au cours de celle-ci. Par exemple, annoncer aux acheteurs que tel lot est vendu à tel prix est un signal.

Outre l'information publique disponible au moment de la vente (description des lots, cours passé des bois), les différents acheteurs commencent la vente avec une certaine quantité d'informations privées que nous appellerons dans la suite leur type. Dans le cas qui nous intéresse, ce type contient entre autres choses de l'information sur les lots obtenue par visite et évaluation privées, de l'information sur les coûts d'exploitation et de l'information sur les débouchés.

Ce qui importe pour l'acheteur, ce n'est pas son type en tant que tel, mais la valeur qu'il accorde au bien. La théorie économique distingue deux situations. On parle de valeur privée si le type d'un acheteur lui suffit pour évaluer le bien en vente. Par exemple, si la seule information importante pour déterminer la valeur d'un lot est l'information sur les coûts d'exploitation et que ces coûts sont connus de l'acheteur, alors la valeur est dite privée. En revanche, si la connaissance du type des autres acheteurs lui fait changer son évaluation, on parle de valeur commune. Nous sommes dans cette situation si l'information importante pour évaluer un lot est, par exemple, le volume réel du lot dont chacun n'a qu'une estimation imparfaite : dans ce cas, deux avis valent mieux qu'un, et chaque acheteur ferait une évaluation plus précise du lot en apprenant, à travers le type d'autres acheteurs, des détails qui lui auraient échappé.

\section{VENTES SÉQUENTIELLES ET COMMUNICATION DE SIGNAUX}

Les ventes de bois par adjudication sous pli cacheté telles qu'elles sont organisées par l'ONF sont des ventes séquentielles. À chaque vente, plusieurs dizaines de lots sont mis en vente les uns après les autres dans un ordre fixé à l'avance par le catalogue des ventes mais dont le point de départ est tiré au sort au début de la vente. Une fois un lot vendu (ou resté invendu), l'organisateur de la vente communique un certain nombre de signaux aux acheteurs : le statut du lot (vendu / invendu), l'identité de l'acheteur, le prix payé par l'acheteur, le nombre et l'identité des soumissionnaires actifs sur ce lot, éventuellement les deuxième et troisième offres nonretenues (sous réserve de garantir l'anonymat) ou encore le prix de retrait. Dans l'organisation actuelle des ventes, ces signaux sont publics, c'est-à-dire communiqués à tous.

Les acheteurs arrivent donc à la vente en connaissant leur type et en ayant en tête une stratégie, c'est-à-dire un plan d'action pour l'ensemble de la vente suivant lequel ils prévoient de réagir de telle ou telle manière aux différents signaux qu'ils recevront au cours de cette vente. Moins ils recevront de signaux au cours de la vente et plus leur stratégie devra être rudimen- 
taire puisqu'elle ne pourra s'adapter à des modifications (non perçues) de l'environnement. Ceci peut être néfaste à l'efficacité de la vente comme au revenu du vendeur.

Pour analyser plus finement le rôle des signaux transmis au cours de la vente, il est utile de décrire leur contenu informationnel et de comprendre leur effet sur les stratégies des acheteurs.

\section{INFORMATION CONTENUE DANS LES SIGNAUX ET IMPACT SUR LES STRATÉGIES}

\section{Information sur le statut de l'acheteur}

Certains signaux sont indiscutablement nécessaires au bon déroulement de la vente parce qu'ils fournissent de l'information à chaque acheteur sur son statut, c'est-à-dire de l'information sur les quantités et les prix pour lesquels l'acheteur est déjà engagé. Un acheteur a certains objectifs et certaines limites de capacité. La quantité de bois déjà obtenue et le prix auquel cette quantité a été achetée est indispensable à l'acheteur pour préparer ses offres. Si aucun signal n'était transmis au cours de la vente, les acheteurs ne sauraient pas la quantité de bois pour laquelle ils sont engagés : la vente ne pourrait pas se dérouler correctement.

\section{Information sur le type des acheteurs}

Certains signaux fournissent de l'information sur les types des acheteurs. Pour mieux appréhender le rôle de cette information, il est nécessaire de revenir à la distinction valeur privée, valeur commune.

Si nous sommes dans une situation à dominante de valeur privée, mieux connaître les types des différents acheteurs a un impact ambigu sur l'efficacité de la vente comme sur le revenu des différentes parties. En effet, mieux connaître le type de ses concurrents devrait permettre à un acheteur d'ajuster sa stratégie en ayant tendance à diminuer le montant de ses offres ; cependant, le fait que son type soit partiellement devenu de l'information publique disponible pour tous peut obliger le même acheteur à être plus agressif pour avoir une chance de gagner un lot (Février, 2003).

Si nous sommes dans une situation à dominante de valeur commune, un nouvel effet intervient. Mieux connaître le type des autres acheteurs permet à chacun d'évaluer plus précisément le lot en vente. En réduisant l'incertitude à laquelle font face les acheteurs, on peut renforcer l'efficacité de la vente et accroître le revenu du vendeur. Cet effet est ce que les théoriciens des enchères appellent une réduction de la « malédiction du vainqueur » (Milgrom, 2004).

\section{Information sur les stratégies des acheteurs}

Enfin certains signaux fournissent de l'information sur les stratégies des acheteurs. Ici, que l'on soit en présence de valeur privée ou de valeur commune, la communication de tels signaux risque d'aider les acheteurs à coordonner leurs stratégies, au détriment du vendeur. Les effets d'une coordination des stratégies des acheteurs sur l'efficacité sont généralement négatifs (Albano et Spagnolo, 2005).

\section{Effets d'anticipation}

Que l'on soit dans une situation de valeur privée ou de valeur commune, le profit réalisé par un acheteur dépend généralement de la quantité d'information privée qu'il possède. Quand les premiers lots sont mis en vente, chaque acheteur anticipe les conséquences de sa stratégie sur 
la probabilité de voir son type devenir partiellement information publique (via les signaux communiqués). Et lorsque les signaux communiqués contiennent principalement de l'information sur les types des acheteurs ayant réalisé les meilleures offres lors de la mise en vente des lots précédents, on peut penser que cela rendra plus risquées les stratégies agressives sur les premiers lots (Jeitschko, 1998).

\section{QUELS SIGNAUX FAUT-IL COMMUNIQUER ?}

En négligeant dans un premier temps les effets d'anticipation, il est utile de retenir les points suivants : la communication de l'information sur le statut des acheteurs a un impact positif sur l'efficacité de la vente, celle de l'information sur les types a un impact positif si l'on retient l'hypothèse de valeur commune et celle de l'information sur les stratégies suivies par les acheteurs a un impact négatif.

Pour évaluer l'intérêt de la communication de tel ou tel signal, il est bon de se servir de la grille de lecture fournie ci-dessus. La difficulté vient évidemment du fait qu'il est généralement impossible de circonscrire l'information fournie par tel ou tel signal. L'identité du vainqueur, par exemple, fournit l'information sur le statut de l'acheteur vainqueur, mais ce signal fournit également de l'information sur la stratégie de ce vainqueur, et sur son type. Il faut donc pour chaque signal déterminer quel sera l'effet dominant.

Il est également important de considérer les effets conjugués des différents signaux. Ainsi un signal communiqué seul peut ne fournir que peu d'information sur les stratégies des acheteurs, mais devenir interprétable dès qu'il est fourni avec un autre signal.

Il faut souligner que nous avons raisonné jusqu'à présent à partir de l'hypothèse que tout signal communiqué l'était de manière publique, c'est-à-dire était communiqué à tous les acheteurs. On peut imaginer transmettre telle ou telle information en choisissant une communication privée des signaux. Le vainqueur d'un lot par exemple pourrait recevoir un signal distinct des autres acheteurs. Ceci est rendu d'autant plus facile quand les ventes sont informatisées. Enfin, une analyse plus fine des enchères de bois devrait sans doute prendre en compte le comportement stratégique du vendeur qui est ici également l'organisateur de la vente (au moins en ce qui concerne le bois des forêts domaniales).

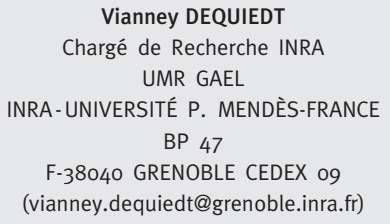

Cette note reprend des réflexions présentées par l'auteur dans le cadre de l'étude “Modes de vente de bois brut et efficacité de la filière-bois française” commanditée par la Direction générale de la Forêt et des Affaires rurales. 


\section{BIBLIOGRAPHIE}

ALBANO (L.), SPAGNOLO (G.). - The Collusive Drawbacks of Sequential Auctions. - Mimeo, 2005.

FÉVRIER (P.). - He Who Must not Be Named. - Review of Economic Design, 8, 2003, pp. 99-119.

JEITSCHKO (T.). - Learning in Sequential Auctions. - Southern Economic Journal, 65, 1998, pp. 98-112.

MILGROM (P.). - Putting Auction Theory to Work. - Cambridge University Press, 2004.

LA COMmUNICATION D'INFORMATIONS RU COURS DES UENTES RUX ENCHËRES DE BOIS PAR ADJUDICATION : CE OUE DIT LA THÉORIE ÉCONOMIOUE [Résumé]

L'objectif de cette note est de proposer un éclairage théorique sur les effets de la communication publique d'informations au cours des ventes aux enchères de bois par adjudication. Elle entend contribuer aux débats entre acheteurs et vendeurs sur ce sujet en présentant une synthèse des recherches menées par les économistes sur cet aspect des ventes aux enchères.

\section{PUBLICIZING INFORMATION RT TIMBER AUCTION SALES - THE UIEU OF ECONOMIC THEORY [Bustract]}

The aim of this paper is to offer some theoretical insight into the effect of publicizing information during timber auctions. The intention is to contribute to the debate between buyers and sellers on this topic by reviewing the research conducted by economists on this aspect of auction sales. 